\title{
Why should we perform hemodynamic ramp test following left ventricular assist device implantation?
}

\section{Teruhiko Imamura*}

Department of Medicine, University of Chicago Medical Center, USA

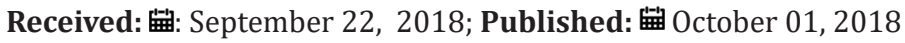

*Corresponding author: Teruhiko Imamura, Department of Medicine, University of Chicago Medical Center, 5841 S. Maryland Avenue Chicago, IL 60637, USA

\begin{abstract}
Left ventricular assist device (LVAD) is a strong therapeutic tool to improve survival in patients with advanced heart failure, whereas not a few patients have potentially abnormal hemodynamics despite LVAD supports. Hemodynamic ramp test, which is recently proposed to undergo following LVAD implantation, is a promising procedure to predict patient clinical outcome by assessing hemodynamic profile and to improve patients' prognosis by optimizing hemodynamics via LVAD speed adjustment. Further prospective randomized control trial is warranted to clearly demonstrate clinical implication of the ramp test.
\end{abstract}

Keywords: Ramp; Hemodynamics; Unloading; Heart Failure

Abbreviations: HF: Heart Failure; LVAD: Left Ventricular Assist Device; PCWP: Pulmonary Capillary Wedge Pressure; CI: Cardiac Index; CVP: Central Venous Pressure

\section{Introduction}

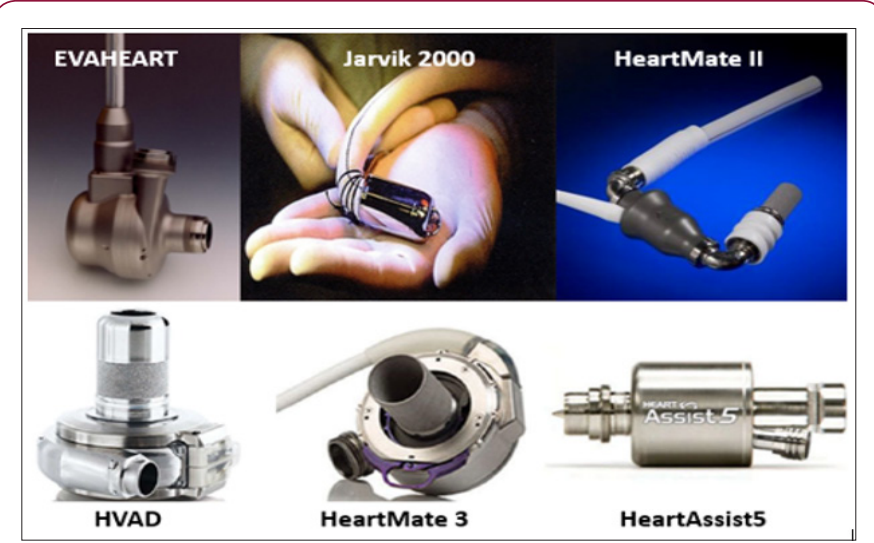

Figure 1: Recently developed left ventricular assist devices (reused from reference 1 with permission).

Owing to the improvement of device technology, more sophisticated management protocol, and cumulating clinical knowledge, actual survival for the patients with advanced heart failure (HF) receiving left ventricular assist device (LVAD) improved dramatically during the recent several decades (Figure 1) [1]. Nevertheless, the rates of re-admission due to device-related comorbidities remain high and unsolved issues thus far [2]. LVAD theoretically normalizes patients' hemodynamics by reducing left ventricular loading and supporting cardiac forward flow. However, our team recently showed that almost half of patients had abnormal hemodynamics following LVAD implantation, despite they were clinically stable outpatients [3]. Optimization of hemodynamics may be a key for overcoming various comorbidities during LVAD support. For the purpose of this, hemodynamic ramp test, which our team recently proposed [3], may be useful for the assessment of hemodynamics and improving clinical outcomes.

What is Hemodynamic Ramp Test?

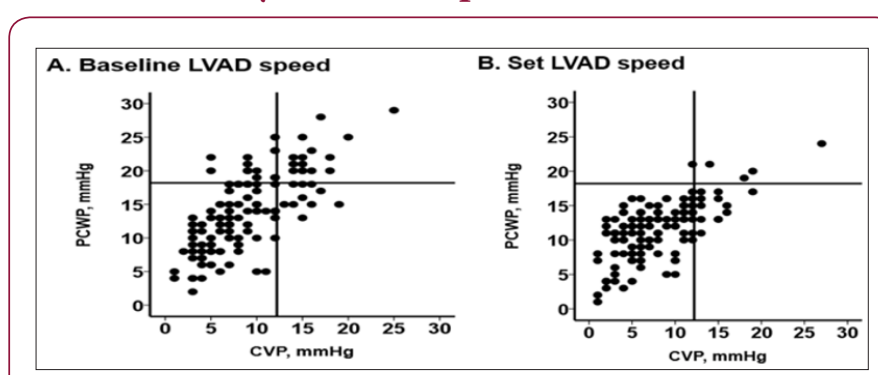

Figure 2: Examples of hemodynamic profiles at baseline LVAD speed (A) and at set LVAD speed (B). CVP, central venous pressure; PCWP, pulmonary capillary wedge pressure; LVAD, left ventricular assist device.

Our team recently proposed a utility of hemodynamic ramp test [3], during which a complete set of hemodynamic parameters is invasively measured by right heart catheterization at each LVAD speed and the LVAD speed is finally adjusted considering the primary goal of central venous pressure (CVP) $<12 \mathrm{mmHg}$, pulmonary 
capillary wedge pressure (PCWP) $<18 \mathrm{mmHg}$, and cardiac index (CI) $>2.2 \mathrm{~L} / \mathrm{min} / \mathrm{m}^{2}$ and the secondary goal of intermittent opening of aortic valve, minimizing mitral valve regurgitation, and appropriate positioning of interventricular septum (Figure 2) $\mathrm{AB}$ is an example of the results, in which CVP and PCWP improve in many patients by LVAD speed adjustment.

\section{Clinical Impact of Optimized Hemodynamics}

Despite promising ability to optimize hemodynamics, the clinical impact of ramp test has remained uncertain. Our team recently demonstrated in the preliminary study, in which optimized hemodynamics that was defined as CVP $<12 \mathrm{mmHg}$, PCWP $<18$ $\mathrm{mmHg}$, and $\mathrm{CI}>2.2 \mathrm{~L} / \mathrm{min} / \mathrm{m}^{2}$, was associated with lower $\mathrm{HF}$ readmission rate. We also demonstrated in another preliminary study that optimized hemodynamics also had an impact on reducing hemocompatibility-related adverse events including bleeding and thrombotic events. Not only the actual values of hemodynamic parameters but also the response to LVAD speed change, which is assessed by the "slope" of each hemodynamic parameter during LVAD speed change [4], may have an association with clinical outcomes. For example, the steeper slopes of PCWP and cardiac output may be associated with better exercise capacity and quality of life [5].

\section{Implication of Ramp Test}

We strongly suggest undergoing hemodynamic ramp test soon after LVAD implantation if not contraindicated, because of the following two main clinical implications of ramp test. The first implication is the assessment of current hemodynamic profile, which is often uncertain when we use only physical examination. We can predict patients clinical outcome considering the results, and also adjust the dose of medications such as diuretics or any other anti-HF agents to stabilize hemodynamics. The second implication is an aggressive optimization of hemodynamics via LVAD speed adjustment. Although we do not have any prospective data, "Ramp-it-Up" ongoing prospective randomized control trial, which compares the clinical outcome between those who received hemodynamic ramp test and those who received only echocardiographic ramp test, may provide us the answer: LVAD speed adjustment during ramp test may improve patients' prognosis. Invasive hemodynamic assessment by using right heart catheterization would be ideal, whereas non-invasive procedures to estimate hemodynamics, including Cardio MEMS and Remote
Dielectric System technology (Figure 3) [1], may be alternatives particularly for repeating hemodynamic assessments.

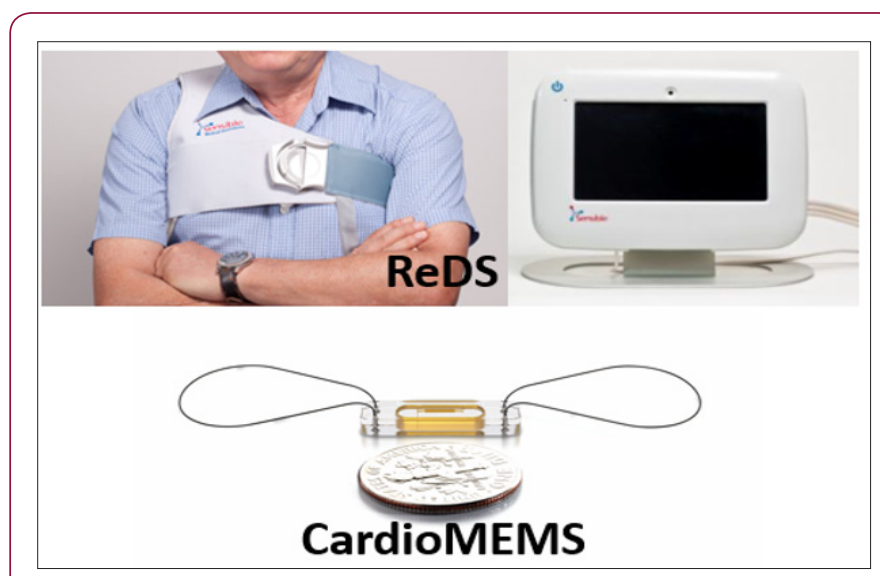

Figure 3: Recently developed non-invasive procedures to estimate hemodynamic parameters ReDS, remote dielectric system (reused from reference 1 with permission).

\section{Conclusion}

Hemodynamic ramp test is a novel procedure to estimate patients' clinical outcomes by assessing hemodynamic profile and rather improve patients' prognosis by LVAD speed adjustment. Prospective randomized control trial is warranted to clearly demonstrate clinical implication of aggressive hemodynamic optimization.

\section{References}

1. Imamura T, Chung B, Nguyen A, Sayer G, Uriel N (2018) Clinical implications of hemodynamic assessment during left ventricular assist device therapy. J Cardiol 71(4): 352-358.

2. Kirklin JK, Pagani FD, Kormos RL, Stevenson LW, Blume ED, et al. (2017) Eighth annual INTERMACS report: Special focus on framing the impact of adverse events. J Heart Lung Transplant 36(10): 1080-1086.

3. Uriel N, Sayer G, Addetia K, Fedson S, Kim GH, et al. (2016) Hemodynamic Ramp Tests in Patients with Left Ventricular Assist Devices. JACC Heart Fail 4(3): 208-217.

4. Nahumi N, Jorde U, Uriel N (2013) Slope calculation for the LVAD ramp test. J Am Coll Cardio 62(22): 2149-2150.

5. Jung MH, Gustafsson F, Houston B, Russell SD (2016) Ramp Study Hemodynamics, Functional Capacity, and Outcome in Heart Failure Patients with Continuous-Flow Left Ventricular Assist Devices. ASAIO J 62(4): 442-446.
ISSN: 2574-1241

DOI: 10.26717/BJSTR.2018.09.001813

Teruhiko Imamura. Biomed J Sci \& Tech Res

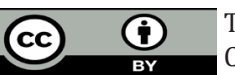

This work is licensed under Creative Commons Attribution 4.0 License

Submission Link: https://biomedres.us/submit-manuscript.php

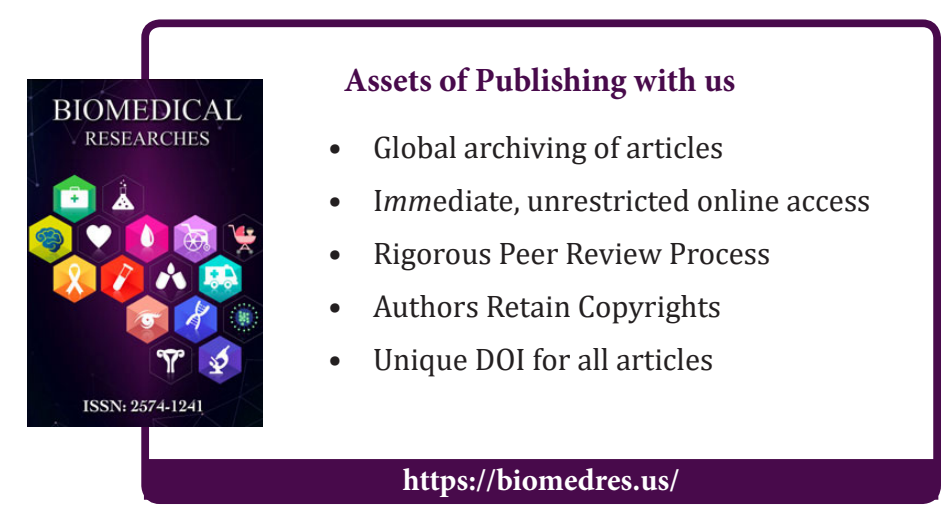

